\title{
VITT, COVID-19 and the Expert Haematology Panel: The story of how the UK responded to emerging cases of vaccine-induced immune thrombocytopenia and thrombosis during the vaccination programme
}

\author{
Authors: Tim Chevassut, ${ }^{A}$ Beverley Hunt $^{B}$ and Sue Pavord ${ }^{C}$
}

The COVID-19 pandemic has resulted in the development of highly effective vaccines that provide hope to the global community for reducing the spread of SARS-CoV-2 and limiting the mortality and morbidity caused by the disease. These vaccines have been produced using differing technologies, taken through clinical trials, and rolled out across the UK at unprecedented speed. However, the recent emergence of rare cases of life-threatening thrombosis in association with thrombocytopenia has threatened to derail one particular vaccine, the Oxford AstraZeneca ChAdOx1 vaccine, upon which many countries are dependent for their vaccination programmes. The story of how this situation has been managed in the UK at the height of the vaccine roll-out represents a remarkable collective endeavour on the part of the haematology community, working closely with other acute medical and surgical professionals within the NHS and the UK health regulatory bodies, to provide rapid expert guidance that has saved lives and helped keep the national vaccination programme on track.

KEYWORDS: VITT, COVID-19, EHP, vaccine, ChAdOx1

DOI: $10.7861 /$ clinmed.2021-0488

The COVID-19 pandemic has resulted in the development of highly effective vaccines that provide hope to the global community for reducing the spread of SARS-CoV-2 and limiting the mortality and morbidity caused by the disease. These vaccines have been produced using differing technologies, taken through clinical trials, and rolled out across the UK at unprecedented

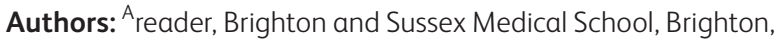
and consultant haematologist, University Hospitals Sussex NHS Foundation Trust, UK; ${ }^{\text {B }}$ professor of thrombosis and haemostasis, King's College, and consultant haematologist, Guy's and St Thomas' NHS Foundation Trust, London, UK; ' Consultant haematologist, Oxford University Hospitals NHS Foundation Trust, Oxford, UK speed. However, the recent emergence of rare cases of lifethreatening thrombosis in association with thrombocytopenia has threatened to derail one particular vaccine, the Oxford AstraZeneca ChAdOx1 vaccine, upon which many countries are dependent for their vaccination programmes. ${ }^{1}$ The story of how this situation has been managed in the UK at the height of the vaccine roll-out represents a remarkable collective endeavour on the part of the haematology community, working closely with other acute medical and surgical professionals within the NHS, and the UK health regulatory bodies, to provide rapid expert guidance that has saved lives and helped keep the national vaccination programme on track.

Vaccine-induced immune thrombocytopenia and thrombosis (VITT) is a rare syndrome affecting around one person in 50,000-100,000 people vaccinated with ChAdOx1. Patients present with serious symptoms $5-30$ days post-vaccination, except in some cases with isolated deep vein thrombosis and pulmonary embolism, where presentation is up to 42 days postvaccination. Patients frequently exhibit very high $\mathrm{D}$-dimer levels, often with low fibrinogen, but the key defining laboratory test is the demonstration of antibodies to platelet factor 4 (PF4). These antibodies, recognised as being similar to those seen in heparininduced thrombocytopenia (HIT), are detected by ELISA-based HIT assays (Lifecodes, Asserachrom and Zymutest); interestingly, they are often not detected by non-ELISA HIT assays, suggesting immunological differences exist between the two conditions. ${ }^{2}$ These anti-PF4 antibodies cause platelet activation leading to thrombocytopenia and acute thrombosis, often in unusual sites. Cerebral venous sinus thrombosis (CVST) accounts for approximately $50 \%$ of presentations and tends to be much more extensive than non-VITT CVST, leading to cerebral oedema, raised intracranial pressure, and secondary intracranial haemorrhage with high mortality, especially if associated with severe thrombocytopenia. Other sites include portal and splanchnic veins, deep vein thrombosis and pulmonary embolism, and arterial ischaemia.

VITT was first described by groups in Norway and Germany among recipients of the $\mathrm{ChAdOx} 1$ vaccine in those countries, leading to temporary cessation of the vaccine within the European Union. 3,4 This was soon followed by a larger series from the UK that defined clinical aspects of VITT and its laboratory diagnosis. ${ }^{5}$ 
Interestingly, VITT-like cases have also been reported in patients who have received the Janssen COVID-19 vaccine (Ad26.COV2.S), causing its temporary cessation in the USA and potentially implicating the adenoviral vector in these vaccines. However, more recently, cases of possible VITT have been reported following the Pfizer BioNTech (BNT162b2) and Moderna (mRNA-1273) vaccines, suggesting the syndrome may be a very rare side-effect of the mRNA COVID-19 vaccines also.

The emergence of VITT as a rare but serious adverse event complicating COVID-19 vaccination prompted urgent action from the haematology community resulting in the rapid formation of an Expert Haematology Panel (EHP) to advise on the investigation and management of suspected cases. Most importantly, the panel has produced living guidance that is hosted on the British Society for Haematology website. ${ }^{6}$ It is based on available evidence and expert opinion in a rapidly evolving situation. The panel has met online almost daily since it was originally convened on 22 March 2021 when cases first started emerging and, to date, has advised on over 300 potential VITT cases, the clinical features of which have recently been published. ${ }^{7}$ Since then, the activities of the EHP have proven far-reaching in providing guidance internationally for managing patients with VITT, and in raising awareness of the syndrome among multidisciplinary primary and secondary care health professionals. Members of the panel have also conducted multi-media interviews with careful balance so as not to jeopardise public confidence in the essential vaccination programme, as has been seen in other countries. However, while VITT remains extremely rare, the diagnosis is potentially catastrophic for affected individuals, often young and healthy, who may have chosen to receive a vaccine for public rather than personal health reasons.

The way the haematology community came together to deal with the emerging VITT 'crisis' is a story that is emblematic of the many remarkable personal efforts made by NHS healthcare professionals throughout the COVID-19 pandemic. Here, we have outlined some of the main achievements of the EHP since its inception.

> To provide mutual and expert opinion to haematologists and other relevant healthcare professionals (including intensivists, neurologists, neurosurgeons, cardiologists, radiologists and pathologists), who have been able to share cases daily via online panel meetings with support provided at other times via phone and email. The rapid dissemination of an awareness of VITT syndrome among haematologists, and best practice for treating affected individuals, was deemed critical for providing local expertise for acute NHS trusts where patients were presenting, often with atypical symptoms.

> To generate live and accessible guidelines highlighting the importance of local haematology support to advise on best treatment based on urgent administration of intravenous immunoglobulin, non-heparin anticoagulation (typically argatroban infusion or fondaparinux) and, in severe cases, transfer to specialist centres for plasma exchange or for neurosurgical intervention. Most VITT patients also received steroid immunosuppression (usually dexamethasone), while rituximab has been used for more refractory cases or those who have relapsed.

> To raise awareness of the VITT syndrome with relevant authorities within the UK, facilitating informed policy decision making, and to work with them as understanding of the condition increased. This has included the chief medical officer
(CMO), Joint Committee for Vaccination and Immunisation (JCVI), Medicine and Healthcare Regulatory Authority (MHRA), Public Health England (PHE), NHS England (NHSE) and the Department of Health. Indeed, the evidence provided by the EHP to these bodies was arguably central to the joint decision by the JCVI and the MHRA to offer the Pfizer vaccine exclusively to individuals below 30 years of age, and subsequently extended to those up to 40 years, where the risk:benefit ratio of vaccination was considered marginal given dwindling numbers of COVID-19 cases across the UK at that time. Confidence in the vaccination programme was deemed critical among these age groups, to whom successful continued roll-out of the vaccination programme is proving central to impeding the spread of the highly transmissible Delta variant of SARS-CoV-2. > To help shape the public message regarding the very small but real risk of vaccination attributable to VITT that the government and $\mathrm{CMO}$ have disseminated to the public via PHE, the MHRA and the JCVI. This distribution of relevant information also included key haematologists within the devolved nations of the UK who could liaise effectively with their respective health authorities.

> To provide timely advice and practical guidance to general practitioners, via the Royal College of General Practitioners, to help address genuine public worry among recipients of the AstraZeneca vaccine who had experienced severe headaches or other symptoms of concern post-vaccination. Balancing the perception of risk from vaccination against the very real risk of death from COVID-19 if unvaccinated against an ongoing challenge in the UK and globally.

> To produce guidelines in collaboration with the Royal College of Emergency Medicine for dealing with worried patients presenting to emergency departments. The VITT situation clearly presented a risk of over-burdening hospital services and radiological scanner capacity. Red flag markers were identified including severity of unexplained symptoms, the timeframe of their onset post-vaccination and the presence of thrombocytopenia on full blood count testing. The guidelines highlighted rapid involvement of a local haematologist for assessing likelihood of diagnosis based on measurement of D-dimers, fibrinogen and urgent PF4 antibody testing by specialist laboratories.

> To collaborate with the neurological community to produce multidisciplinary guidelines on optimal neurological management of CVST (involving neurologists, neurosurgeons, neuroradiologists and intensivists who often have different approaches for dealing with these critically ill patients). The focus here was directed primarily at immediate intervention with intravenous immunoglobulins to block platelet activation combined with therapeutic anticoagulation to limit thrombus propagation.

> To identify a particularly poor prognostic group and recommend escalation of treatment to early plasma exchange for those patients with clinical deterioration and severe thrombocytopenia who have an extremely high risk of mortality from intracranial haemorrhage and cerebral oedema. Decisions regarding surgical decompression and/or thrombectomy, as well as when to administer platelets and plasma products preoperatively, require rapid multidisciplinary discussions.

> To collaborate with NHS Blood and Transplant to help generate guidelines relevant to the donation of organs from deceased VITT patients whose organs might be non-viable due to small 
vessel thromboses and whose blood might carry the potential for inducing iatrogenic VITT in the recipient.

> To develop written patient information for individuals diagnosed with VITT, including avoidance of repeat vaccination with the AstraZeneca vaccine, and to provide appropriate recommendations to physicians regarding follow-up management and optimal anti-coagulation and/or anti-platelet therapy.

> To engage the help of NHSE in monitoring the national shortage of intravenous immunoglobulin and to ensure that supplies were safeguarded and prioritised to be immediately available at all UK hospitals for any VITT patients who required them.

> To develop National Institute for Health and Care Excellence guidelines that would serve as an international reference for doctors elsewhere in the world, particularly resource-poor countries who are heavily reliant on the Oxford AstraZeneca vaccine. $^{8}$

> To establish a national commitment for psychological and social support for patients and their families involving the CMO, NHSE and PHE, as well as working closely with Thrombosis UK who have been providing support to individuals with VITT, their families and the bereaved.

> To collaborate with the MHRA and facilitate and conduct relevant scientific studies focused on understanding the pathogenic mechanism of VITT that will be required for improved treatment and even prevention of this disease in the future, including vaccine design and identification of risk factors. These studies have included whole genome sequencing, immunological analysis, platelet activation assays and in vitro drug testing, and have already identified novel strategies for switching off platelet activation resulting in candidate therapies to be tested in future international trials.

In summary, with the emergence of a new life-threatening condition, the haematology community have risen to the challenge to define it, explore best treatments and engage with all areas within clinical care to ensure that if a patient is diagnosed with VITT anywhere in the UK, they will receive up-to-date consensus management and follow-up. This has required many hours of altruistic input, and we salute all those involved who have given their time freely.

\section{References}

1 Cines DB, Bussel JB. SARS-CoV-2 vaccine-induced immune thrombotic thrombocytopenia. N Engl J Med 2021;384:2254-6.

2 Platton S, Bartlett A, MacCallum P et al. Evaluation of laboratory assays for anti-platelet factor 4 antibodies after ChAdOx1 nCOV-19 vaccination. J Thromb Haemost 2021;19:2007-13.

3 Schultz NH, Sørvoll IH, Michelsen AE et al. Thrombotic thrombocytopenia after ChAdOx1 nCov-19 vaccination. N Engl ] Med 2021;384:2124-30.

4 Greinacher A, Thiele T, Warkentin TE et al. Thrombotic thrombocytopenia after ChAdOx1 nCov-19 vaccination. N Engl J Med 2021;384:2092-101.

5 Scully M, Singh D, Lown R et al. Pathologic antibodies to platelet factor 4 after ChAdOx1 nCoV-19 vaccination. N Engl J Med 2021;384:2202-11.

6 British Society for Haematology. Guidance produced by the Expert Haematology Panel (EHP) focussed on Vaccine induced Thrombosis and Thrombocytopenia (VITT). BSH, 2021. https://bs-h.org.uk/about-us/news/guidance-produced-by-the-experthaematology-panel-ehp-focussed-on-vaccine-induced-thrombosisand-thrombocytopenia-vitt

7 Pavord S, Scully M, Hunt B] et al. Clinical features of vaccineinduced immune thrombocytopenia and thrombosis. N Engl J Med 2021, in press (DOI: 10.1056/NEJMoa2109908).

8 National Institute for Health and Care Excellence. COVID-19 rapid guideline: vaccine-induced immune thrombocytopenia and thrombosis (VITT): NICE guideline [NG200]. NICE, 2021. www.nice.org. uk/guidance/ng200

Address for correspondence: Dr Sue Pavord, Oxford University Hospitals NHS Foundation Trust, Oxford, UK. Email: Sue.Pavord@ouh.nhs.uk

Twitter: @SuePavord 